\title{
POINCARÉ INEQUALITY FOR ABSTRACT SPACES
}

\author{
Alireza Ranjbar-Motlagh
}

\begin{abstract}
The Poincaré inequality is generalised to metric-measure spaces which support a strong version of the doubling condition. This generalises the Poincare inequality for manifolds whose Ricci curvature is bounded from below and metric-measure spaces which satisfy the measure contraction property.
\end{abstract}

\section{INTRODUCTION}

In this article, the Poincare inequality is extended to quite general abstract metricmeasure spaces, that is, a metric space $(X, d)$ with a measure $\mu$ (see $[7,12,20]$ for the basic definitions). Recently, many authors have studied the Sobolev spaces for maps over abstract spaces. See for instance Heinonen [12] and the references therein. A Poincare type inequality is one of the main theorems that we expect to be satisfied (and meaningful) for abstract spaces. The Poincaré inequality means, roughly speaking, that the $L^{p}$-norm of a function can be controlled by the $L^{p}$-norm of its derivative (up to a universal constant). It is well-known that the Poincaré inequality implies the Sobolev and isoperimetric inequalities in doubling and smooth spaces; see $[11,19]$. Also, Cheeger [5] provided applications of Poincaré type inequalities for metric-measure spaces.

Heinonen and Koskela [13]. Bourdon and Pajot [2], Laakso [17], Hanson and Heinonen [9], Garofalo and Nhitid [8], and Lanconelli and Morbidelli [18], Semmes [24], Strum [25] and Kuwae and Shioya [16] studied the Poincaré type inequality on abstract spaces. The author $[\mathbf{2 1}]$ introduced a version of the triangle comparison property on metric spaces and the uniform doubling condition on metric-measure spaces such that the Poincaré type inequality is valid for such spaces. For more work about this topic on Riemannian manifolds, groups, and graphs see $[19,23,26]$. See also $[11]$ and the references therein.

The main purpose of this article is to introduce a strong version of the doubling condition on metric-measure spaces which implies the Poincaré inequality (see [6] for the basic properties of doubling spaces). In particular, this implies that if the domain of functions is a Riemannian manifold whose Ricci curvature is bounded from below, then

Received 5th July, 2004

The author would like to thank the Research Council of Sharif University of Technology and Institute for Studies in Theoretical Physics and Mathematics for support.

Copyright Clearance Centre, Inc. Serial-fee code: 0004-9727/05 $\$ A 2.00+0.00$. 
a Poincaré type inequality is valid for such manifolds (on the bounded domains). As a consequence, we obtain the Sobolev and isoperimetric inequalities for such manifolds which generalises a well-known result of Buser [3]. Here, we use the definition of (extended) upper gradient (see Definition 2.1) on abstract spaces so that in the Poincaré inequality the norm of the derivative remains meaningful. This article is an extension of the author's work [21]. Moreover, the definition of the strongly doubling condition, Definition 2.4, generalises the definition of weak measure contraction property in [25, Definition 4.1] and weak measure contraction property of Bishop-Gromov type in [15] (see also Remark 2.2 and 2.5).

\section{Definitions}

In this section, we introduce a sufficient condition on abstract spaces which implies the Poincare inequality. This condition is a strong version of the doubling condition on metric-measure spaces and it can also be interpreted as a version of the conclusion of the Bishop-Gromov volume comparison theorem in Riemannian geometry; for instance see [19].

First, we recall and generalise some basic definitions relating to metric-measure spaces. For simplicity, we assume that all metric spaces are second countable and locally compact and all (outer) measures are Radon (see [7] for definitions). We denote the closed ball of radius $r>0$ with centre at $x$ by $B(x, r)$ (in a metric space $(X, d)$ ).

Let $(X, d, \mu)$ be a metric-measure space. It is said that $X$ is a locally doubling space, if for every $R>0$ there is $C_{R}>0$ such that

$$
\mu(B(x, 2 r)) \leqslant C_{R} \mu(B(x, r)),
$$

for all $x \in X$ and $0<r<R$. The constant $C_{R}$ is called the (local) doubling constant of $X$. A locally doubling space $X$ is called a doubling space, if there is $C>0$ such that $C_{R} \leqslant C$, for all $R>0$.

A metric-measure space $(X, d, \mu)$ is called a locally uniformly doubling space (see [21]), if the condition

$$
\mu(B(x, 2 r)) \leqslant C_{R} \mu(B(y, r)),
$$

holds for all $x, y \in X$ and $0<r<R$ whenever $d(x, y) \leqslant 2 R$. The constant $C_{R}$ is called the (local uniform) doubling constant of $X$. A locally uniformly doubling space $X$ is called a uniformly doubling space, if there is $C>0$ such that $C_{R} \leqslant C$, for all $R>0$.

A metric-measure space $(X, d, \mu)$ is called locally Ahlfors regular, if for every $0<R<\operatorname{diam}(X)$ there are $K_{R}>0$ and $n>0$ such that

$$
K_{R}^{-1} r^{n} \leqslant \mu(B(x, r)) \leqslant K_{R} r^{n},
$$


for all $x \in X$ and $0<r<R$. The number $n$ is called the dimension of $X$. A locally Ahlfors regular space $X$ is called Ahlfors regular, if there exists positive number $K$ such that $K_{R} \leqslant K$, for all $0<R<\operatorname{diam}(X)$.

It is easy to see that every (locally) Ahlfors regular metric-measure space is a (locally) uniformly doubling space.

A metric space $(X, d)$ is called a geodesic space, if for every $x, y \in X$, there is a geodesic $\gamma_{x, y}:[0,1] \longrightarrow X$ (with the velocity $d(x, y)$ ) from $x$ to $y$, that is, $d\left(\gamma_{x, y}(s), \gamma_{x, y}(t)\right)=|s-t| d(x, y), \gamma(0)=x$ and $\gamma(1)=y$. Next, we recall the definition of (extended) upper gradient which is a generalisation of the norm of gradient over smooth functions.

Definition 2.1: Let $(X, d, \mu)$ be a metric-measure space. Let $X$ be a geodesic space. Suppose that there is a measurable function $\Phi: X \times X \times[0,1] \longrightarrow X$ such that

$$
\Phi(x, y, s)=\Phi(y, x, 1-s)=\gamma_{x, y}(s),
$$

where $\gamma_{x, y}$ is a geodesic from $x$ to $y$. Let $u$ be a (real-valued) function on $X$. A nonnegative Borel measurable function $g$ is said to be an (extended) upper gradient for $u$, if the following condition holds:

$$
|u(x)-u(y)| \leqslant d(x, y) \int_{0}^{1} g(\Phi(x, y, s)) d s,
$$

for almost everywhere $(x, y) \in X \times X$.

REMARK 2.2. The definition of (extended) upper gradient (as in Definition 2.1) is slightly weaker than [13, Definition 2.9]. Compare [5, Definition 2.8]. Moreover, in Definition 2.1, instead of the geodesics $\gamma_{x, y}$, we can consider $K$-quasi geodesics, that is, if the condition

$$
d\left(\gamma_{x, y}(s), \gamma_{x, y}(t)\right) \leqslant K|s-t| d(x, y),
$$

holds for some $K>0$.

REMARK 2.3. In a quite general setting, the map $\Phi$ is (can be constructed to be) measurable. In fact, by a construction which is due to Kuratowski and Ryll-Nardzewski $[1$, p. 90], we are able to construct a measurable selection.

Now, we introduce the definition of strongly doubling metric-measure spaces which can be interpreted as a generalisation of the conclusion of Bishop-Gromov volume comparison theorem in Riemannian geometry. Compare [21, Proposition 2.7].

Definition 2.4: Let $X$ and $\Phi$ be as in Definition 2.1. We say that $X$ is locally strongly doubling, if for every $R>0$ there is $b_{R}>0$ such that for almost everywhere $x \in X$, and any Borel measurable (open) subset $A \subset B(x, R)$, we have

$$
\mu(\{z \in B(x, R): \Phi(x, z, t) \in A\}) \leqslant b_{R} \mu(A),
$$


for all $1 / 2 \leqslant t \leqslant 1$. We say that $X$ is strongly doubling, if there exists $\bar{b}>0$ such that $b_{R} \leqslant \bar{b}$, for all $R>0$.

REMARK 2.5. Similarly to $[\mathbf{2 5}, \mathbf{1 5}]$, we are able to extend Definition 2.4 to (locally) strongly doubling spaces with exceptional set.

It is clear that every (locally) strongly doubling space is a (locally) doubling space as well. The author does not know the exact relation between uniformly doubling and strongly doubling conditions over metric-measure spaces. But, in a metric space with bounded geometry (see [21, Definition 2.2]), the uniformly doubling condition implies the strongly doubling condition, see [21, Proposition 2.7]. Therefore, this article generalises the results of [21]. Moreover, Definition 2.4 (see also Remark 2.2 and 2.5) generalises the definitions of the weak measure contraction property in [25, Definition 4.1] and the weak measure contraction property of Bishop-Gromov type in [15]. See also [25, Proposition 4.5]. Therefore, this article recovers the Poincare type inequality proved in $[\mathbf{2 5}$, Theorem $6.3]$ and [16, Theorem 4.2] (for the energy norm, we use the upper gradient).

\section{Generalised Change of Variables}

In this section, we state and proof a generalised change of variables formula for functions over strongly doubling metric-measure spaces. We start this section with the following simple fact. As was mentioned before, for simplicity, we assume that all metric spaces are second countable and locally compact and all (outer) measures are Radon.

Lemma 3.1. Let $(X, d, \mu)$ be a metric-measure space. Let $E$ be a measurable subset of $X$ of positive and finite measure, that is, $0<\mu(E)<\infty$. Let $\varepsilon$ be a positive number. Then, there exist a (closed) subset $F \subset E$ and $\delta_{0}>0$ such that

$$
\begin{gathered}
\mu(E \backslash F) \leqslant \varepsilon, \\
\mu\left(\bigcup_{x \in F} B(x, \delta)\right) \leqslant 2 \mu(E),
\end{gathered}
$$

for all $0<\delta \leqslant \delta_{0}$.

Proof: Let $\varepsilon>0$. There is a compact subset $F \subset E$ such that $\mu(E \backslash F) \leqslant \varepsilon$. For $\delta>0$, define

$$
F_{\delta}:=\{z \in X: d(z, F) \leqslant \delta\} .
$$

Note that, for $\delta$ small enough, $F_{\delta}$ is compact (by the Lebesgue number lemma) and also

$$
\lim _{\delta \rightarrow 0} \mu\left(F_{\delta}\right)=\mu(F) .
$$

Therefore, by choosing $\delta$ small enough, we have

$$
\mu\left(\bigcup_{x \in F} B(x, \delta)\right) \leqslant \mu\left(F_{\delta}\right) \leqslant 2 \mu(E) .
$$

This completes the proof of lemma. 
THEOREM 3.2. (Generalised change of variables) Let $(X, d, \mu)$ be a locally strongly doubling metric-measure space. Let $g$ be a non-negative Borel measurable function on $X$. Let $\Phi$ be as in Definition 2.1. Then, for almost everywhere $a \in X$, we have

$$
\int_{B(a, R)}\left(\int_{1 / 2}^{1} g(\Phi(a, y, s)) d s\right) d \mu(y) \leqslant M \int_{B(a, R)} g(z) d \mu(z)
$$

where $R>0$ and $M$ is a constant which depends on $b_{R}$ (as in Definition 2.4).

Proof: Without loss of generality, we can assume that the map $h(y, t):=\Phi(a, y, t)$ is measurable. Let $\varepsilon$ be a small positive number and let $1 / 2 \leqslant s \leqslant 1$. Since that $X$ is a locally doubling space, there exist a finite sequence, say $\left\{x_{\alpha}\right\}$, of points $B(a, R s)$ and a finite sequence, say $\left\{A_{\alpha}\right\}$, of Borel measurable subsets $B(a, R s)$ with the following conditions:

1. $B\left(x_{i}, \varepsilon / 2\right) \cap B\left(x_{j}, \varepsilon / 2\right)=\phi$, for all $i \neq j$.

2. $B(a, R s)=\bigcup_{\alpha} A_{\alpha}$.

3. $A_{\alpha} \subset B\left(x_{\alpha}, \varepsilon\right)$.

4. $A_{i} \cap A_{j}=\phi$, for all $i \neq j$.

Define a finite sequence of subsets $B(a, R)$ as the following:

$$
E_{\alpha}:=\left\{w \in B(a, R): h(w, s) \in A_{\alpha}\right\} .
$$

By Lemma 3.1, for any positive number $\bar{\varepsilon}$, there is a (closed) subset $F_{\alpha}$ of $E_{\alpha}$ (if $\mu\left(E_{\alpha}\right)$ $>0)$ and a positive number $\delta_{\alpha}$ such that $\mu\left(E_{\alpha} \backslash F_{\alpha}\right) \leqslant \bar{\varepsilon}$ and $\mu\left(\bigcup_{x \in F_{\alpha}} B(x, r)\right) \leqslant 2 \mu\left(E_{\alpha}\right)$, for all $0<r<\delta_{\alpha}$. Suppose that $0<\delta \leqslant \min \delta_{\alpha}$. Since that $X$ is a locally doubling

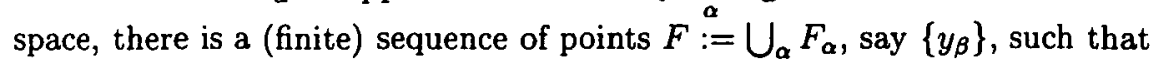

1. $B\left(y_{i}, \delta / 2\right) \cap B\left(y_{j}, \delta / 2\right)=\phi$, for all $i \neq j$.

2. $F \subset \bigcup_{\beta} B\left(y_{\beta}, \delta\right)$.

Therefore, there exist a finite sequence, say $\left\{Y_{\alpha}\right\}$, of subsets such that $Y_{\alpha} \subset F_{\alpha}$ and numbers $\delta, \eta \in] 0, \varepsilon\left[\right.$ (depending on $F_{\mathrm{a}}$ 's) such that

1. $B(z, \delta / 2) \cap B(w, \delta / 2)=\phi$, for all $z, w \in Y:=\bigcup_{\alpha} Y_{\alpha}$, and $z \neq w$.

2. $B(a, R) \backslash G \subset \bigcup_{y \in Y} B(y, \delta)$, where $G$ is a measurable subset of $B(a, R)$ such that $\mu(G) \leqslant \eta$.

3. $\mu\left(\bigcup_{z \in Y_{\alpha}} B(z, \delta)\right) \leqslant 2 \mu\left(E_{\alpha}\right)$. 
Then, we have

$$
\begin{aligned}
\sum_{z \in Y} \mu(B(z, \delta)) g(h(z, s)) & \leqslant C_{R} \sum_{z \in Y} \mu(B(z, \delta / 2)) g(h(z, s)) \\
& \leqslant C_{R} \sum_{\alpha}\left(\sum_{z \in Y_{\alpha}} \mu(B(z, \delta / 2)) g(h(z, s))\right) \\
& \leqslant C_{R} \sum_{\alpha} \mu\left(\bigcup_{z \in Y_{\alpha}} B(z, \delta / 2)\right) \max _{z \in Y_{\alpha}}\{g(h(z, s))\} \\
& \leqslant 2 C_{R} \sum_{\alpha} \mu\left(E_{\alpha}\right) \max _{z \in Y_{\alpha}}\{g(h(z, s))\} \\
& \leqslant 2 C_{R} b_{R} \sum_{\alpha} \mu\left(A_{\alpha}\right) \max _{z \in Y_{\alpha}}\{g(h(z, s))\} \\
& \leqslant 2 C_{R} b_{R} \sum_{\alpha} \mu\left(B\left(x_{\alpha}, \varepsilon\right)\right) \max _{z \in Y_{\alpha}}\{g(h(z, s))\}
\end{aligned}
$$

where $C_{R}$ is a constant which depends on the (local) doubling constant of $X$ (or $b_{R}$ ). Hence, for every $\varepsilon>0$, there exist a finite subset $Y$, a sequence $\left\{x_{\alpha}\right\}$, a measurable subset $G$ and numbers $\delta, \eta \in] 0, \varepsilon$ ( (with the above properties) such that

$$
\sum_{z \in Y} \mu(B(z, \delta)) g(h(z, s)) \leqslant M \sum_{\alpha} \mu\left(B\left(x_{\alpha}, \varepsilon / 2\right)\right) \sup _{x \in B\left(x_{\alpha}, \varepsilon\right)}\{g(x)\},
$$

where $M$ is a constant which depends on $b_{R}$. It is enough to show

$$
\int_{B(a, R)} g(h(y, t)) d \mu(y) \leqslant M \int_{B(a, R+\varepsilon)} g(z) d \mu(z),
$$

for all $1 / 2 \leqslant t \leqslant 1$ and $\varepsilon>0 *$, where $M$ is a constant which depends on $b_{R}$. The proof of above fact is similar to the proof of [21, Lemma 3.1]. But, for the sake of completeness we provide the details of proof here. The complete proof of lemma is based on the following statements:

(a) If (3.2) is valid for bounded measurable functions $g$, then it is valid for all measurable functions $g$.

(b) If both functions $g(\cdot)$ and $g(h(\cdot, t))$ are continuous, then (3.2) is valid for such $g$.

(c) If (3.2) is valid for continuous functions $g$, then it is valid for measurable functions $g$.

(d) (3.2) is valid for all continuous functions $g$.

Proof OF (a): Suppose that (3.2) holds for all bounded functions. Apply (3.2) to the bounded functions

$$
g_{N}(x):=g(x) \chi_{\{y: g(y) \leqslant N\}}(x)
$$


where $\chi$ denotes the characteristic function and $N$ is a positive integer. Letting $N \rightarrow \infty$. This implies that (3.2) holds for $g$. From now on, we assume that $0 \leqslant g(x) \leqslant K$, for all $x$ and some number $K$.

Proof of (b): This is an immediate consequence of (3.1). Note that we have assumed that all metric spaces are locally compact, therefore, every continuous function on a closed (and bounded) ball is uniformly continuous. Also, it is easy to see that a locally doubling space is complete if and only if every closed (and bounded) ball is compact.

Proof of (c): Suppose that (3.2) holds for all continuous functions. Let $g$ be a measurable function. Let $\delta>0$. By the Luzin theorem (see $[\mathbf{2 2}$, p. 55]) there is a continuous function $u$ such that

$$
\begin{aligned}
0 & \leqslant u(x) \leqslant K, \quad \forall x, \\
\mu(A) & \leqslant \delta,
\end{aligned}
$$

where $A:=\{x \in B(a, R): u(x) \neq g(x)\}$. By assumptions, we have

$$
\begin{aligned}
\mu(\{y \in B(a, R): u(h(y, t)) \neq g(h(y, t))\}) & =\mu(\{y \in B(a, R): h(y, t) \in A\}) \\
& =\mu(\{y \in B(a, R): \Phi(a, y, t) \in A\}) \\
& \leqslant b_{R} \mu(A) \\
& \leqslant b_{R} \delta,
\end{aligned}
$$

where $1 / 2 \leqslant t \leqslant 1$ is a fixed number. Then, since (3.2) holds for $u$, we have

$$
\int_{B(a, R)} u(h(y, t)) d \mu(y) \leqslant M \int_{B(a, R+\varepsilon)} u(z) d \mu(z)
$$

and

$$
-2 K\left(b_{R} \delta\right)+\int_{B(a, R)} g(h(y, t)) d \mu(y) \leqslant M\left(\int_{B(a, R+\varepsilon)} g(z) d \mu(z)+K \delta\right) .
$$

Letting $\delta \rightarrow 0$. This implies $g$ satisfies (3.2).

Proof of (d): We prove (3.2) for continuous function $g$ (and $0 \leqslant g \leqslant K$ ). By the Luzin theorem, for every $\tau \in] 0, \varepsilon$, there is a continuous function $v$ such that

$$
\begin{aligned}
0 & \leqslant v(x) \leqslant K, \quad \forall x, \\
\mu\left(U^{c}\right) & \leqslant \tau,
\end{aligned}
$$

where $U:=\{y \in B(a, R): v(y)=g(h(y, s))\}$ and $U^{c}:=B(a, R) \backslash U$. Suppose that $\varepsilon, \delta$, $\eta, F, Y, G$ and $\left\{x_{\alpha}\right\}$ are as before. We can choose the sequence $\left\{y_{\beta}\right\}$ (as before) with the following extra conditions:

1. $y_{j} \in F \cap U$, for all $j$. 
2. $F \cap U \subset \bigcup_{\beta} B\left(y_{\beta}, \delta\right)$.

3. $\mu[B(a, R) \backslash(F \cap U)] \leqslant 2 \varepsilon$.

Note that sequences $\left\{x_{\alpha}\right\}$ and $\left\{y_{\beta}\right\}$ satisfy (3.1). We have

$$
\begin{aligned}
\int_{F \cap U} v(y) d \mu(y) & \leqslant \sum_{z \in Y} \int_{B(z, \delta)} v(y) d \mu(y) \\
& \leqslant \sum_{z \in Y} \mu(B(z, \delta)) \sup _{B(z, \delta)} v .
\end{aligned}
$$

Then, we obtain

$$
\begin{aligned}
\sum_{\alpha} \mu\left(B\left(x_{\alpha}, \varepsilon / 2\right)\right) \inf _{B\left(x_{\alpha}, \varepsilon\right)} g & \leqslant \sum_{\alpha} \mu\left(B\left(x_{\alpha}, \varepsilon / 2\right)\right) \inf _{B\left(x_{\alpha}, \varepsilon / 2\right)} g \\
& \leqslant \sum_{\alpha} \int_{B\left(x_{\alpha}, \varepsilon / 2\right)} g(z) d \mu(z) \\
& \leqslant \int_{B(a, R+\varepsilon)} g(z) d \mu(z) .
\end{aligned}
$$

Because $v$ is uniformly continuous on closed (and bounded) balls, we have

$$
\begin{aligned}
\sum_{z \in Y} \mu\left(B(z, \delta) \sup _{B(z, \delta)} v\right. & \leqslant \sum_{z \in Y} \mu(B(z, \delta))[v(z)+\lambda] \\
& \leqslant \lambda+\sum_{z \in Y} \mu(B(z, \delta)) g(h(z, s))
\end{aligned}
$$

where $\lambda \geqslant 0$ depends on $\varepsilon, g, v, B(a, R)$ and $b_{R}$. Moreover, $\lambda \rightarrow 0$ as $\varepsilon \rightarrow 0$. Therefore, we have (and using (3.1))

$$
\begin{aligned}
\int_{F \cap U} v(y) d \mu(y) & \leqslant \lambda+\sum_{z \in Y} \mu(B(z, \delta)) g(h(z, s)) \\
& \leqslant \lambda+M \sum_{\alpha} \mu\left(B\left(x_{\alpha}, \varepsilon / 2\right)\right) \sup _{B\left(x_{\alpha}, \varepsilon\right)} g \\
& \left.\leqslant \lambda+M \sum_{\alpha} \mu\left(B\left(x_{\alpha}, \varepsilon / 2\right)\right) \inf _{B\left(x_{\alpha}, \varepsilon\right)} g+\lambda\right] \\
& \leqslant \lambda+M \sum_{\alpha}^{\alpha} \mu\left(B\left(x_{\alpha}, \varepsilon / 2\right)\right) \inf _{B\left(x_{\alpha}, \varepsilon\right)} g \\
& \leqslant \lambda+M \int_{B(a, R+\varepsilon)} g(y) d \mu(y) .
\end{aligned}
$$


This implies that

$$
\begin{aligned}
\int_{B(a, R)} g(h(y, s)) d \mu(y) & =\int_{B(a, R) \backslash(F \cap U)} g(h(y, s)) d \mu(y)+\int_{F \cap U} g(h(y, s)) d \mu(y) \\
& =\int_{B(a, R) \backslash(F \cap U)} g(h(y, s)) d \mu(y)+\int_{F \cap U} v(y) d \mu(y) \\
& \leqslant K \mu[B(a, R) \backslash(F \cap U)]+\int_{F \cap U} v(y) d \mu(y) \\
& \leqslant 2 K \varepsilon+\int_{F \cap U} v(y) d \mu(y) \\
& \leqslant 2 K \varepsilon+\lambda+M \int_{B(a, R+2 \varepsilon)} g(z) d \mu(z) .
\end{aligned}
$$

Letting $\varepsilon \rightarrow 0$ (note that $\delta, \eta, \tau \in] 0, \varepsilon[$ ), then we obtain

$$
\int_{B(a, R)} g(h(y, s)) d \mu(y) \leqslant M \int_{B(a, R)} g(z) d \mu(z),
$$

for all $1 / 2 \leqslant s \leqslant 1$. This completes the proof of theorem.

\section{PoINCARÉ INEquality}

In this section, we prove the following weak $(1,1)$-Poincaré type inequality. The proof is based on the generalised change of variables formula, Theorem 3.2.

THEOREM 4.1. (Weak Poincaré inequality) Let $(X, d, \mu)$ be a locally strongly doubling metric-measure space. Let $g$ be an (extended) upper gradient for a measurable function $u$ on $X$. Then, we have

$$
f_{B(a, R)} f_{B(a, R)}|u(x)-u(y)| d \mu(x) d \mu(y) \leqslant M R f_{B(a, 3 R)} g(z) d \mu(z),
$$

where $a \in X, R>0$, and $M$ is a constant which depends on $b_{3 R}$ (as in Definition 2.4).

PROof: See the proof of [21, Theorem 3.3].

REMARK 4.2. Let notations and assumptions be as in Theorem 4.1. If the ball $B(a, R)$ is convex, then we have (see [21, Remark 3.5])

$$
f_{B(a, R)} f_{B(a, R)}|u(x)-u(y)| d \mu(x) d \mu(y) \leqslant M R f_{B(a, R)} g(z) d \mu(z) .
$$

Also, If we impose a mild condition on $X$, we are able to obtain a strong $(p, p)$-Poincaré inequality, see [10].

Now, we provide some examples of spaces which satisfy the assumptions of Theorem 4.1 . 
Propositron 4.3. Let $(X, d, \mu)$ denote the Euclidean space $\mathbf{R}^{n}$ with the usual Euclidean metric $d$, and arbitrary doubling (Borel) measure $\mu$. Then, the following conditions are equivalent:

(i) The space $(X, d, \mu)$ is (locally) strongly doubling.

(ii) The space $(X, d, \mu)$ is (locally) uniformly doubling.

(iii) The space $(X, d, \mu)$ is (locally) Ahlfors regular of dimension $n$.

ProOF: It is an immediate consequence of [21, Proposition 2.5].

Proposition 4.4. Let $\left(M^{n}, g\right)$ be a complete $n$-dimensional Riemannian manifold such that

$$
\underset{M}{\operatorname{Ric}} \geqslant-(n-1) \lambda,
$$

for some $\lambda \geqslant 0$. Then

(i) $M$ is a locally strongly doubling metric-measure space. In fact, we can choose

$$
b_{R}:=2^{n} e^{2(n-1) \sqrt{\lambda} R} .
$$

(ii) ([3] and [4, p. 288]. Let $S$ be a smooth hypersurface in $B(x, R)$. Suppose that $S$ divides $B(x, R)$ into two parts, denoted by $D_{1}$ and $D_{2}$. Then, we have

$$
\min \left\{\operatorname{Vol}\left(D_{1}\right), \operatorname{Vol}\left(D_{2}\right)\right\} \leqslant C \operatorname{Area}(S),
$$

where $C$ is a constant which depends on $n, \lambda$ and $R$.

PROOF:

(i) The proof follows by inspection in the proof of Bishop-Gromov volume comparison theorem (for example see [19, Corollary 2.3]). See also [14, Theorem 1.4.1] and [23].

(ii) The proof is a straightforward consequence of the equivalence of Sobolev and isoperimetric inequalities (see [19, Theorem 9.2]).

\section{REFERENCES}

[1] J.P. Aubin and A. Cellina, Differential inclusions, Die Grundlehren der mathematischen Wissenschaften 264 (Springer-Verlag, New York, 1984).

[2] M. Bourdon and H. Pajot, 'Poincaré inequalities and quasiconformal structure on the boundary of some hyperbolic buildings', Proc. Amer. Math. Soc. 127 (1999), 2315-2324.

[3] P. Buser, 'A note on the isoperimetric constant', Ann. Sci. École Nor. Sup. 15 (1982), 213-230.

[4] I. Chavel, Riemannian geometry - A modern introduction (Cambridge Univ. Press, Cambridge, 1993).

[5] J. Cheeger, 'Differentiability of Lipschitz functions on metric measure spaces', Geom. Funct. Anal. 9 (1999), 428-517. 
[6] R. Coifman and G. Weiss, 'Extensions of Hardy spaces and their use in analysis', Bull. Amer. Math. Soc. 83 (1977), 569-645.

[7] L.C. Evans and R.F. Gariepy, Measure theory and fine properties of functions, Studies in Adv. Math. (CRC Press, Boca Raton, Florida, 1992).

[8] N. Garofalo and D.-M. Nhieu, 'Isoperimetric and Sobolev inequalities for Carnot-Carathéodory spaces and existence of minimal surfaces', Comm. Pure Appl. Math. 49 (1996), 1081-1144.

[9] B. Hanson and J. Heinonen, 'An $n$-dimensional space that admits a Poincaré inequality but has no manifold points', Proc. Amer. Math. Soc. 128 (2000), 3379-3390.

[10] P. Hajlasz and P. Koskela, 'Sobolev meets Poincaré', C. R. Acad. Sci. Paris Ser. I Math. 320 (1995), 1211-1215.

[11] P. Hajłasz and P. Koskela, 'Sobolev Met Poincaré', Mem. Amer. Math. Soc. 145 (2000).

[12] J. Heinonen, Lectures on analysis on metric spaces (Springer-Verlag, New York, 2001).

[13] J. Heinonen and P. Koskela, 'Quasiconformal maps in metric spces with controlled geometry', Acta Math. 181 (1998), 1-61.

[14] N. Korevaar and R. Schoen, 'Global existence theorems for harmonic maps to non-locally compact spaces', Comm. Anal. Geom. 5 (1997), 333-387.

[15] K. Kuwae and T. Shioya, 'On generalized measure contraction property and energy functionals over Lipschitz maps', Potential Anal. 15 (2001), 105-121.

[16] K. Kuwae and T. Shioya, 'Sobolev and Dirichlet spaces over maps between spaces', (preprint).

[17] T.J. Laakso, 'Ahlfors $Q$-regular spaces with arbitrary $Q>1$ admitting weak Poincaré inequality', Geom. Funct. Anal. 10 (2000), 111-123. With erratum, Geom. Funct. Anal. 12650 (2002).

[18] E. Lanconelli and D. Morbidelli, 'On the Poincaré inequality for vector fields', Ark. Mat. 38 (2000), 327-342.

[19] P. Li, Lecture notes on geometric analysis, Lecture Notes Series 6 (Seoul National University, Research Institute of Mathematics, Global Analysis Research Center, Seoul, 1993).

[20] A. Ranjbar-Motlagh, Analysis on metric-measure spaces, (Ph. D. Thesis) (New York University, New York, 1998).

[21] A. Ranjbar-Motlagh, 'A note on the Poincaré inequality', Studia Math. 154 (2003), 1-11.

[22] W. Rudin, Real and complex analysis, (3rd edition) (McGraw-Hill Book Co., 1987).

[23] L. Saloff-Coste, Aspects of Sobolev-type inequalities, LMS Lecture Note Series 289 (Cambridge Univ. Press, Cambridge, 2002).

[24] S. Semmes, 'Finding curves on general spaces through quantative topology, with applications to Sobolev and Poincaré inequalities', Selecta Math. 2 (1996), 155-295.

[25] K.T. Sturm, 'Diffusion processes and heat kernels on metric spaces', Ann. Probab. 26 (1998), 1-55.

[26] N.T. Varopoulos, L. Saloff-Coste and T. Coulhon, Analysis and geometry on groups (Cambridge Univ. Press, Cambridge, 1992). 
Department of Mathematical Sciences

Sharif University of Technology

P.O. Box 11365-9415

Tehran

Iran

e-mail: ranjbarm@sharif.edu 